\title{
Abtragung von Knorpel- und Knochengewebe mittels fasergeführtem Excimerlaserstrahl
}

\author{
W. NEU, M. DRESSEL \\ Laser-Laboratorium Göttingen e.V., Im Hassel 21, 3400 Göttingen
}

R. JAHN

Unjversitäts-Krankenhaus Hamburg-Eppendorf, Abteilung Unfallchirurgic (Direktor: Prof.Dr.K.H. Jungbluth), Martinistr. 52, D-2000 Hamburg 20

U. GRZESIK

Heracus Quarzglas GmbH, Postfach 1554, D-6450 Hanau

\section{Laseranwendung in der Unfallchirurgie}

Die Anwendung des Lasers als Schneidinstrument in der Medizin beschränkt sich seit nunmehr fast drei Jahrzehnten ausschließlich auf Weichgewebe. Chirurgische Fachgebiete, wie z.B. dic Unfallchirurgie, bei denen vorwiegend Trennverfahren (Sägen, Bohren. Mcißeln) am biologischen Hartgewebe (Knorpel, Knochen) vorgenommen werden müssen, waren bislang von der Anwendung des Lasers ausgenommen. Das Schneiden von Hartgewebe erfordert hohe Applikationsenergien, die sich bei Anwendung herkömmlicher, meist kontinuicrlicher Laser aus dem nahen und fernen infraroten Spektralbereich (Nd:YAG-, $\mathrm{CO}_{2}$-Laser) bislang in heilungshemmende thermische Wirkungen umsetzten, gekennzeichnet durch breite Nekrose- und Karbonisationszonen insbesondere im Knochengewebe [1]. Die ersten Versuche, gepulste Laser aus dem UV-Bereich (Excimerlaser) cinzusetzen zeigten zwar nur geringe thermische Nebenwirkungen, doch scheiterten sie vor allem an der geringen Transmissionsfāhigkeit von Lichtleitfasern [2] und damit der geringen Abtragsraten.

Bei der Ablation von Gewebe mit kurzgepulsten Excimerlasern erfolgt die Materialabtragung nicht mehr ausschlicßlich durch photothermische Wirkung, sondern indem auch molekulare Bindungen aufgrund der hohen Photonenenergie des Lasers aufgebrochen werden. Die entstehenden gasförmigen Ablationsprodukte und Materialtrümmer werden dabei explosionsartig aus dem Bestrahlungsvolumen herausgeschleudert [3]. Bei diesem Abtragungsmechanismus treten nur sehr geringe thermische Nebenwirkungen auf, da die Laserwellenlānge stark absorbiert, damit verbunden nur eine geringe Eindringtiefe erreicht wird und zusätzlich die Bestrahlungsdauer aufgrund des kurzen Laserpulses im Vergleich zu thermischen Diffusionszeiten klein ist. Die anfänglich errcichten Abtragungsgeschwindigkeiten erwiesen sich für Operationszwecke als zu niedrig. Darüber hinaus ist ein Einsatz als unfallchirurgisches Instrument im Sinne einer minimal-invasiven Anwendung, insbesondere in bisher schwer zugānglichen oder komplikationstrāchtigen Regionen, erst durch die Möglichkeit der Laserstrahlführung über optische Lichtleitfasern denkbar. Daher und aufgrund der für eine effiziente Ablation notwendigen starken Absorption der Laserstrahlung kann zur Zeit allein der XeCl-Excimerlaser mit der Wellenlänge $\lambda=308 \mathrm{~nm}$ eingesetzt werden. Durch die Verwendung von Taperfasern als flexible Lichtleiter konnten beachtliche Abtragraten und 
Schneidgeschwindigkeiten erreicht werden [4]. Die grundsātzlichen Wechselwirkungenmechanismen bei der Ablation biologischen Hartgewebes, wie z.B. Knochen oder Knorpel, sind bisher jedoch nur wenig erforscht.

\section{Material und Methode}

Zur systematischen Bestimmung und Optimierung der Ablationsratc, d.h. der Operationsgeschwindigkeit, an Knochen- und Knorpelgewebe wurde der in Abbildung 1 gezcigte Versuchsaufbau verwendet. Wir setzten drei unterschiedliche XeCl-Excimerlasersysteme

\section{experimental setup}

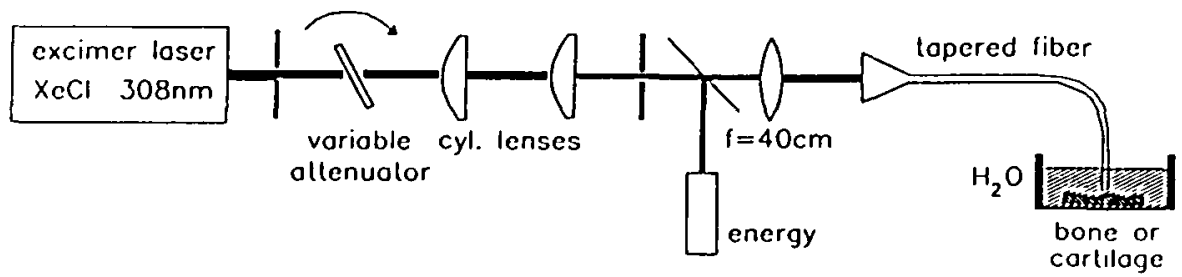

Abbildung 1: Versuchsaußbau zur Ablation biologischer Hartgewebe mit XeCl-Excimerlaserstrahlung $(\lambda=308 \mathrm{~nm})$ geführt durch Taperfasern.

$(\lambda=308 \mathrm{~nm})$ mit Pulsbrciten von $28 \mathrm{~ns}, 60 \mathrm{~ns}$ und 250-300 ns ein. Die Laserencrgie wurde über Taperfasern übertragen, die cine unkritische Transmission sehr hoher Pulsenergien bis zu $250 \mathrm{~mJ}$ zulassen [2,1]. Dic verwendeten Taperfasern hatten einen Kerndurchmesser von $200 \mu \mathrm{m}, 400 \mu \mathrm{m}$, $600 \mu \mathrm{m}$ und $1000 \mu \mathrm{m}$. Das konische Einkoppelteil der Taperfasern ist jeweils ctwa im Verhältnis 1:10 aufgewcitet. Dic Laserparameter variierten über folgende Bereiche: Encrgie am distalen Ende der Faser von $20 \mathrm{~mJ} \cdot 70 \mathrm{~mJ}$; Repetitionsrate $10-100 \mathrm{~Hz}$. Dic Ablationen wurden als Schnitte und Bohrungen an Rippenknochen und Meniski frisch geschlachteter Rinder vorgenommen. In mehr als 400 Einzelapplikationen wurden dabei die Wechselwirkungen zwischen den verschiedenen Laserparametern und dem Gewebe untersucht.

\section{Ergebnisse und Diskussion}

Schon in den crsten Experimenten bestätigte sich die faserschützende Wirkung längerer Pulsbreiten, im Vergleich zur Standardpulsbreite von $\Delta \tau=28$ ns. Dic Abhängigkeit der Oberflächenzerstörschwelle, dic proportional zur Quadratwurzel der Laserpulsbreite steigt, erlaubt die Transmission höherer Energien bei längeren Pulsen bzw. eine Schonung der Faseraustrittsfäche bei konstanter Pulsenergie. Es zeigte sich, daß es bei der Standardpulsbreite von $\Delta \tau=28 \mathrm{~ns}$ ab ciner Ausgangsenergie von $40 \mathrm{~mJ}$ fast regelmāBig zur Zerstörung der Fascrendflāchen kommt. 


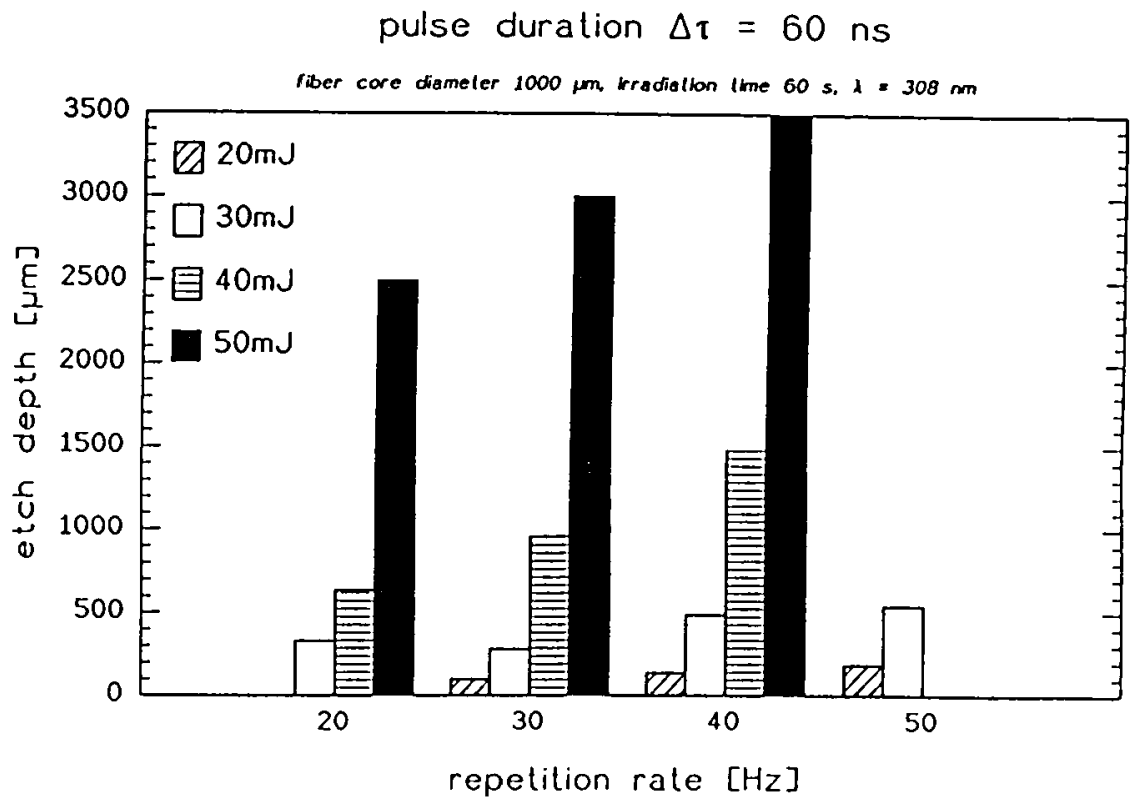

Abbildung 2: Bohrlochtiefen von Rinderknochen in Wasser bei eincr Bestrahlungszeit von $60 \mathrm{~s}$. XeCl-Excimerlaser $\lambda=308 \mathrm{~nm}$, Pulsdauer $\Delta \tau=60 \mathrm{~ns}$, Taperfaser mit Kerndurchmesser $1000 \mu \mathrm{m}$

Applikationen mit dem Langpulslaser $(\Delta \tau=250-300 \mathrm{~ns})$ sind auch noch bei Energien von bis zu $50 \mathrm{~mJ}$ ohne cine Zerstörung der Faserendfläche möglich. Interessante Ergebnisse brachte der XeClExcimerlaser mit einer Pulsbreite von $\Delta \tau=60$ ns (s. Abbildung 2). Bis zu ciner Repetitionsrate von $40 \mathrm{~Hz}$ waren die Bohrungen mitunter um das 3-4fache tiefer als bei Anwendung des Langpulslasers. Dies deutet darauf hin, daß der entscheidende Parameter der Ablation die Leistungsdichte, d.h. dic Energic/Pulsdauer, ist. Bei einer weiteren Steigerung der Repetitionsrate aul $50 \mathrm{~Hz}$ und der Energie über $30 \mathrm{~mJ}$ wird die Faser derzeit noch zerstōrt.

Die gesetzten Bohrungen und Schnitte mit allen verwendeten Excimerlasersystemen sind insgesamt charakterisicrt durch gleichmäßige, saubere Ränder und auch in der Ticfe zeigen sich kcine Karbonisationen. Wir errcichen cine Ablationsrate von 3-5 $\mu \mathrm{m} / \mathrm{Puls}$, entsprechend $0,08 \mathrm{~mm} / \mathrm{s}$ Bohrbzw. Schnittgeschwindigkeit im Kinochengewebe. Diese Werte weisen auf die prinzipielle Möglichkeit einer "athermischen" linochengewebsdurchtrennung hin. An den untersuchten Rindermeniski erziclten wir Schnitt- und Bohrgeschwindigkeiten von bis zu $6 \mathrm{~mm} / \mathrm{s}$.

\section{Ausblick}

Bemerkenswert ist, daß, obwohl im Rahmen dieser Versuche mit steigenden Transmissionsenergien gearbeitet wurde, der Sättigungsbereich der Ablatıonsrate für Knochen noch nicht erreicht worden ist. Aufbauend auf diesen Erfahrungen kann nach weiteren technischen Verbesserungen der Fasern und der Applikatoren damit gerechnet werden, daß die Ablationsraten auch für Knochen 
noch wesentliche Steigerungen erfahren und damit intraoperativ Abtragungen von Exostosen, dystopen Verknöcherungen oder die Ausräumung von osteitischen Knochenherden möglich werden. Osteotomien großer Röhrenknochen beim Erwachsenen sollten für diesen Laser nicht die Zielstellung sein.

\section{Literatur:}

1. Dinkelaker F: Dic $\mathrm{CO}_{2}$-Laser-Osteotomie Habilitationsschrift Chirurgische Klinik und Poliklinik der Freien Universität Berlin 1989

2. Dressel M, Neu W, Gerhardt H: Quarzglasfasern für die Übertragung von Excimerlaserpulsen hoher Leistung. Laser und Optoelektronik 22(5): 76-81 (1990)

3. Neu W, Nyga R, Tischler C, Haase KK, Karsch KR: Ultrafast imaging of vascular tissuc ablation by a XeCl excimer laser. SPIE Vol. 1425: 37-44 (1991)

4. Jahn R, Dressel M, Fabian H, Gerhardt H, Kesper J, Klein KF, Langendorff HU, Neu W, Sowada U, Jungbluth KH: Excimerlaser und Taperfaser - ein effizientes Instrument für die Ablation von Knochen- und Knorpelgewebe. Laser in medicine and surgery 6(2): 77-80 (1990)

Wir danken den Firmen Lambda Physik, Göttingen und der Heraeus Quarzglas GmbHI, Hanau für die freundliche Unterstūtzung dieser Versuche. 